\title{
COVID-19 Vaccine, Asymptomatic Cases, Risk Communication and Community Engagement in Sub-Saharan Africa
}

\author{
David Houéto ${ }^{1 *}$, Ferdinand Daga ${ }^{2}$, Alexandre Timée ${ }^{2}$, Romulus Honhonou ${ }^{3}$, Maurice Agonnoudé ${ }^{1}$ \\ ${ }^{1}$ School of Public Health (ENATSE), University of Parakou, Parakou, Benin \\ ${ }^{2}$ Institute of nursing and obstetrics Education (IFSIO), University of Parakou, Parakou, Benin \\ ${ }^{3}$ School of Medicine (FM), University of Parakou, Parakou, Benin \\ Email: *dhoueto@yahoo.fr
}

How to cite this paper: Houéto, D., Daga, F., Timé, A., Honhonou, R. and Agonnoudé, M. (2021) COVID-19 Vaccine, Asymptomatic Cases, Risk Communication and Community Engagement in Sub-Saharan Africa. Open Journal of Preventive Medicine, 11, 71-79. https://doi.org/10.4236/ojpm.2021.113007

Received: February 10, 2021

Accepted: March 14, 2021

Published: March 17, 2021

Copyright $\odot 2021$ by author(s) and Scientific Research Publishing Inc. This work is licensed under the Creative Commons Attribution International License (CC BY 4.0).

http://creativecommons.org/licenses/by/4.0/

\begin{abstract}
African region will probably be the last region in COVID-19 vaccine uptake. But that shouldn't be a problematic issue if the emphasis is maintained on the role Risk Communication and Community Engagement (RCCE) can play in controlling the COVID-19 pandemic through the adoption of preventive measures. We used the issue of asymptomatic cases to illustrate the importance that should still be given to preventive measures such as hand washing, mask wearing, physical and social distancing, knowing that vaccines have never been $100 \%$ effective. Also with the issue of variants in COVID-19, the vaccine couldn't be considered as a panacea. Lessons from the Nordic countries are strong arguments to put emphasis on preventive measures in the region.
\end{abstract}

\section{Keywords}

COVID-19 Vaccine, Asymptomatic, Preventive Measures, Risk

Communication and Community Engagement

\section{Background}

The epidemic of COVID-19 infections that broke out in China in late 2019 has rapidly grown and cases have been reported worldwide including the African Region (Figure 1). The time from illness onset in a primary case (infector) to illness onset in a secondary case (infectee) is still unclear in order to understand the turnover of case generations and transmissibility of the disease. On the ground, countries adopted as a crucial part of control efforts, isolation of confirmed and suspected cases, and identification of contacts. With the situation of 


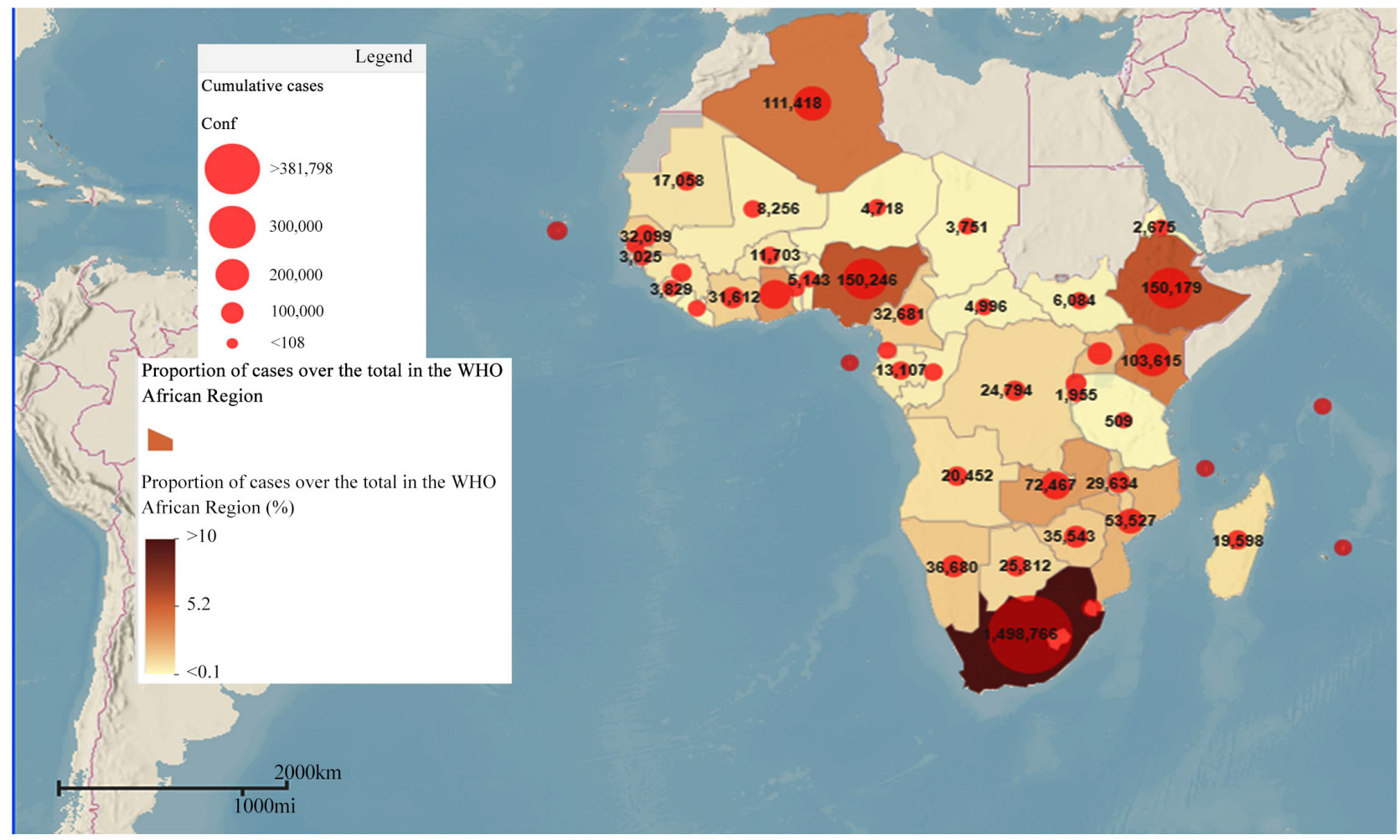

Figure 1. COVID-19 in the WHO African Region, situation as of February $19^{\text {th }} 2021,1: 00$ AM, cumulative cases. (Countries affected: 47; Cumulative cases: 2,744,432; Deaths: 24,464). Source:

https://who.maps.arcgis.com/apps/opsdashboard/index.html\#/0c9b3a8b68d0437a8cf28581e9c063a9

community transmission, another question for consideration is a group of asymptomatically infected individuals, which may propagate the virus and impede infection control knowing that access to COVID-19 vaccine is still on the way coming in the African region [1].

What is an asymptomatic case and how it can be taken into account by Risk Communication and Community Engagement (RCCE) professionals for better interaction with communities though the perspective of vaccine is on the way in the region?

\section{Definitions}

In general, the asymptomatic case is asymptomatic someone who carries a germ (virus or bacteria) in his body but never develops any symptoms related to the specific disease caused by that germ. Asymptomatic of COVID-19 are people who carry active virus in their body but never develop any symptoms (they are tested positive with no development of symptoms). In other words, there is no fever, no gastrointestinal issues, no breathing issues, no coughing, etc. in link with COVID-19. As one might imagine, it's hard to figure out when someone has a disease but shows no signs of it. Some cases of asymptomatic carriers have been confirmed by finding and testing people who were in close contact with COVID-19 patients. For those who tested positive without symptoms, follow-up exams confirmed that about $25 \%$ continued to show no signs [2]. That means 
some of this asymptomatic who tested positive and developed symptoms during follow-up exams were pre-symptomatic.

The pre-symptomatic are people who have been infected and are incubating the virus but don't yet show symptoms. With COVID-19, after infection, symptoms might not develop for five to six days-or even two weeks (detection of virus prior to the development of symptoms) [3]. In other words, the time between catching the virus and showing symptoms is called the pre-symptomatic phase. In general, when it comes to infection transmission, people appear to be most infectious right around the time when symptoms start. However, there is evidence, from testing and modeling studies that suggests people who are pre-symptomatic can definitely transmit COVID-19, most likely in the one to three days before they start showing symptoms, according to the World Health Organization (WHO) [4].

So far, according to $\mathrm{WHO}$, pre-symptomatic is a much more common category than asymptomatic. About $75 \%$ of people who test positive without showing symptoms turn out to be pre-symptomatic, displaying coughing, fatigue, fever and other signs of COVID-19 in a later follow-up exam [2]. Among these pre-symptomatic cases, there are many of them who are in fact very mildly symptomatic.

Are very mildly symptomatic, people who feel a little unwell from a COVID-19 infection but continue to come in close contact with others? COVID-19 is then transmitted by people with whom symptoms remain mild. So people who continue to frequent communal and public places with a light cough or mild fever may unintentionally spread COVID-19 in the early days of symptom onset. Even when a person's own symptoms remain mild, others they infect can become very sick.

\section{The Magnitude of the COVID-19 Asymptomatic Cases}

The amount of people who are circulating in the population without knowing they've been infected with the coronavirus

No one knows for sure, and for the moment lots of the evidence is anecdotal. In general, having an infection without any symptoms is common. In the case of the flu for instance, estimates are that anywhere from $5 \%$ to $25 \%$ of infections occur with no symptoms. For the most part, symptoms are actually a side effect of fighting off an infection. It takes a little time for the immune system to rally that defense, so some cases are more aptly considered pre-symptomatic rather than asymptomatic. For the COVID-19, estimates suggest that about $80 \%$ of people with COVID-19 have a mild or asymptomatic disease, 14\% have severe disease, and 6\% are critically ill [5], implying that symptom-based control is unlikely to be sufficient unless these cases are only lightly infectious. Japanese researchers estimate that more than $90 \%$ of cases are undetected [6]. So, given that asymptomatic transmission has been documented, utmost caution is urged [7] [8]. Also, cases of COVID-19 among nursing home residents, choir groups and 
families fuel a growing concern about people who are infected, yet feel generally "healthy" and go about their daily lives, giving the virus to friends, family members and strangers without knowing that they themselves have it.

\section{Contagiousness of an asymptomatic COVID-19 person}

The onset and duration of viral shedding and the period of infectiousness for COVID-19 are not yet well known. Most recently it has been shown that high levels of the virus are present in respiratory secretions during the "pre-symptomatic" period that can last days to more than a week prior to the fever and cough characteristic of COVID-19 [9]. This ability of the virus to be transmitted by people without symptoms is a major reason for the pandemic and that should lead to a rigorous adoption of preventive measures with a risk communication and community engagement (RCCE) strategy. The virus also spreads through normal exhalations that can carry tiny droplets containing the virus. A regular breath may spread the virus several feet or more. Spread could also come from surfaces, such as a doorknob or a grocery cart handle, that are contaminated with the coronavirus by an infected person's touch. No matter what, if one has been exposed to someone else with COVID-19, that person should self-quarantine for the entire 14-day incubation period. Even if that person feels fine, he is still at risk of spreading the coronavirus to others.

Proportion of asymptomatic and pre-symptomatic cases responsible for COVID-19 transmission

The vital load detected in an asymptomatic person was similar to that in symptomatic people [10]. This means asymptomatic cases may be as likely to transmit infection as symptomatic ones. Further, the highest viral load is early in the illness when symptoms are mild [11]. A recent modeling paper suggests that in China before the lockdown, undiscovered cases, main people with "mild, limited or no symptoms", were less infectious than known cases but were still possibly responsible for $79 \%$ of transmission, because so many of them continued to congregate or travel while contagious [12]. Studies from Singapore [11] and China [13] suggest that pre-symptomatic cases account for $6 \%$ to $13 \%$ of transmission. In addition, children and young people may be a reservoir for asymptomatic, mildly symptomatic or pre-symptomatic transmission [14]. Given the potential for asymptomatic infection, to prevent sustained community transmission, quarantine is necessary. Further, testing only high-risk travelers or contacts may miss infectious cases. Serological tests will be helpful for better understanding the extent of asymptomatic transmission [15]. Even though there is still much to learn about silent spreading, the concerns about this mode of transmission give more weight to the advice we've been hearing all along: Keep a 6-foot distance from others, wash hands often and wipe down surfaces. We do not need to wait for symptoms to protect those around us [16].

Asymptomatic COVID-19 infection appears to be common and will continue to complicate efforts to get the pandemic under control, that's why some measures have to be taken and reinforced by the RCCE professionals. 


\section{Measures to Be Reinforced by the Rcce Professionals}

\section{Importance of preventive measures}

Examples from the Nordic countries like Denmark suggest changing the way we look at health to shift to a more preventive approach and highlight the need for a new social contract between the individual, community and the organizations. As of May 1, 2020, while the entire world is being hard hit by a novel emerging coronavirus, Denmark had a total of 9311 cases and 460 deaths, whereas other countries such as Switzerland, roughly similar in size and population and only $1000 \mathrm{~km}$ distance from Denmark, have already accumulated three times more cases and deaths. Lessons from Denmark show the importance of trust in the health system in particular and the government in general, and culture. Danish people have few physical contacts. They do give hugs, but only to close family members or friends; they do not shake hands; they do not kiss each other to say hello; and generally speaking, they like to keep a decent social distance on public transportation and in other public places [17].

\section{Vaccine uptake}

In general, vaccines provide direct protection by reducing susceptibility to disease or infection and thus reduce the number of people infected in a population or their infectiousness. But this does not mean that we are free of risk. As good as they are, vaccines are not $100 \%$ effective, and that is the case also with COVID-19 vaccines. Even if the vaccine does work properly, it's possible that one could be protected against severe infection, but still has an asymptomatic infection. Also with COVID-19, there is the issue of variants that can be more transmissible than the viruses that were circulating before. And they may not always be protected against the vaccines that were developed several months ago. It's then still possible to spread the virus after being vaccinated. So, frequent hand washing, masking up, physical and social distancing remains critical, especially for the African region [18]. Although the African region is blessed with a young population $(60 \%$ to $70 \%$ are under 30$)$ it is challenged by fragile health systems and endemic diseases. As cases begin to increase sharply, Africa has an uncertain future with COVID-19. Regarding the vaccine coverage in the African region, as of 25 January 2021, more than 68 million doses of COVID-19 vaccines have been administered in 56 countries across the world. Among these countries are four from Africa: Egypt, Guinea, Morocco and Seychelles [19].

While trying to engage with communities, knowing that we are all affected by the COVID-19 pandemic, RCCE professionals must have on hand some Q\&A regarding the preventive measures in order to deal with uncertainties and contribute to the decision-making that protects them.

\section{When should one suspect COVID-19?}

People should suspect that they might have COVID-19 if they are experiencing fever, cough and/or shortness of breath. These symptoms, however, are also symptoms of other illnesses. For example, in the African region, fever and cough are also symptoms of flu. But also, fever can be the expression of malaria or any 
other infectious disease. So, it is important to pay attention to symptoms but to also know they will not necessarily mean that one has COVID-19. One may need to have a health professional advice.

\section{When to call or see a health professional?}

With fever, cough and/or shortness of breath, one can call either the local hotline or a health professional. Make sure to not just show up; it is very important to call first. Most health professionals will be able to give advice on what to do in order to help the caller to know his/her status. If need be, Doctors' offices will have ways to isolate someone who potentially has COVID-19 before directing that person to a treatment center. Doctor's office will likely ask the person screening questions on the phone and will provide instructions about what to do on arrival or direct the caller to an appropriate health facility in charge of COVID-19.

When someone has a runny nose and sniffles, could this be coronavirus?

A runny nose or nasal congestion would not likely be COVID-19. But if the person doubts, he/she should call the local hotline or a health professional to be sure he/she is safe.

What to expect from a hotline staff member or at a health facility?

Hotline staff is trained to help understand the symptoms and the history behind them in order to direct a caller for an appropriate action to take. There is a need to stay calm and explain!

When going to the health facility, put on a face mask before. The health care provider will test for COVID-19, especially if he/she concludes after examination that there are some risk factors. That would include having traveled to a country or area with sustained person-to-person transmission, or having been in contact with someone with COVID-19.

Why one should wear a face mask though he/she is not COVID-19 positive?

They're a big reason public health officials have suggested everyone should wear face masks. A COVID-19 patient is releasing coronavirus through the droplets he/she is spraying out from cough or sneeze. To avoid being infected by droplets (through mouth or nose) coming from someone whose status is unknown, wearing a face mask is a good protection.

\section{Why teleworking is recommendable whenever it is possible?}

Teleworking could curb the COVID-19 epidemic because working from home pays a double dividend during a pandemic. First, it can help to limit the spread of the highly contagious coronavirus. These support organizations' efforts to limit travel and major public events, and more assertive efforts by governments in badly affected regions to restrict population movement altogether [20]. Second, restricting travel and canceling events have substantial costs at a time when businesses are already dealing with absences due to illnesses. Allowing people to work from home can help cut some of these losses [21].

What is the role of border control, quarantine and isolation?

Travel is the major vector for transmission of infections globally, so border 
control measures, including restriction of travel (lockdown), will reduce transmission. Travel bans and quarantine are proven interventions, and especially critical for infections with pre-symptomatic or asymptomatic transmission as it is the case with COVID-19 [22]. Travel restrictions, quarantine of "healthy" people who have been in contact with a case or are otherwise high risk for becoming ill, and isolation of people with infection (whether symptomatic or not) are key interventions for COVID-19 control.

\section{Rationale of the eradication attempt}

The public health goal is to prevent the epidemic from becoming sustained, or if that is not possible, to delay it and reduce the total number of cases using all available interventions. Delay provides time for preparedness, expansion of quarantine and isolation capacity, and shortens the time to availability of a possible vaccine. If nothing is done, countries would face a larger and more prolonged epidemic that would cause greater stress on the health systems. As such, the response strategy should persist with all feasible measures for as long as possible. That's where the role of RCCE is crucial in engaging communities in adopting and maintaining preventive measures. Our capacity for intensive care unit and isolation beds may rapidly be exhausted, as well as stockpiles of personal protective equipment and other supplies [23].

\section{Conclusion}

Asymptomatic cases in COVID-19 are an important aspect of its control. Although the progress in COVID-19 vaccine uptake in the African region may be the ultimate goal to control the pandemic as it is the case in the developed countries, it shouldn't prevent us from the importance to be given to RCCE to reinforce preventive measures. African region still needs to continue washing frequently hands, wearing facial masks, keeping physical and social distances for effective control of COVID-19 even when vaccine comes.

\section{Conflicts of Interest}

The authors declare no conflicts of interest regarding the publication of this paper.

\section{References}

[1] Zhou, P., Yang, X.L., Wang, X.G., et al. (2020) A Pneumonia Outbreak Associated with a New Coronavirus of Probable Bat Origin. Nature, 579, 270-273. https://doi.org/10.1038/s41586-020-2012-7

[2] WHO (2020) COVID-19-Virtual Press Conference-01 April 2020. World Health Organization, Geneva.

https://www.who.int/docs/default-source/coronaviruse/transcripts/who-audio-emer gencies-coronavirus-press-conference-full-01apr2020-final.pdf?sfvrsn $=573 \mathrm{dc140} 2$

[3] Lauer, A.S., Grantz, H.K., Bi, Q., et al. (2020) The Incubation Period of Coronavirus Disease 2019 (COVID-19) from Publicly Reported Confirmed Cases: Estimation and Application. Annals of Internal Medicine, 172, 577-582.

https://doi.org/10.7326/M20-0504 
[4] WHO (2020) Coronavirus Disease 2019 (COVID-19) Situation Report-73. Data as Reported by National Authorities by 10:00 CET 2 April 2020. World Health Organization, Geneva.

https://www.who.int/docs/default-source/coronaviruse/situation-reports/20200402sitrep-73-covid-19.pdf?sfvrsn=5ae25bc7_6

[5] European Centre for Disease Prevention and Control (2020) Latest Assessment on COVID-19 from the European Centre for Disease Prevention and Control (ECDC). Eurosurveillance, 25, Article No. 2002271. https://doi.org/10.2807/1560-7917.ES.2020.25.8.2002271

[6] Nishiura, H., Kobayashi, T., Yang, Y., et al. (2020) The Rate of under Ascertainment of Novel Coronavirus (2019-nCoV) Infection: Estimation using Japanese Passengers' Data on Evacuation Flights. Journal of Clinical Medicine, 9, 419. https://doi.org/10.3390/jcm9020419

[7] Bai, Y., Yao, L., Wei, T., et al. (2020) Presumed Asymptomatic Carrier Transmission of COVID-19. Journal of the American Medical Association, 323, 1406-1407. https://doi.org/10.1001/jama.2020.2565

[8] Tong, Z.D., Tang, A., Li, K.F., et al. (2020) Potential Pre-Symptomatic Transmission of SARS-CoV-2, Zhejiang Province, China, 2020. Emerging Infectious Diseases Journal, 26, 1052-1054. https://doi.org/10.3201/eid2605.200198

[9] Gandhi, M., Yokoe, S.D. and Havlir, V.D. (2020) Asymptomatic Transmission, the Achilles' Heel of Current Strategies to Control Covid-19. The New England Journal of Medicine, 382, 2158-2160. https://doi.org/10.1056/NEJMe2009758

[10] Zou, L., Ruan, F., Huang, M., et al. (2020) SARS-CoV-2 Viral Load in Upper Respiratory Specimens of Infected Patients. The New England Journal of Medicine, 382, 1177-1179. https://doi.org/10.1056/NEJMc2001737

[11] Wei, W.E., Li, Z., Chiew, C.J., et al. (2020) Pre-Symptomatic Transmission of SARS-CoV-2-Singapore, January 23-March 16, 2020. Morbidity and Mortality Weekly Report, 69, 411-415. https://doi.org/10.15585/mmwr.mm6914e1

[12] Li, R., Pei, S., Chen, B., et al. (2020) Substantial Undocumented Infection Facilitates the Rapid Dissemination of Novel Coronavirus (SARS-CoV-2). Science, 368, 489-493. https://doi.org/10.1126/science.abb3221

[13] Du, Z., Xu, X., Wu, Y., et al. (2020) Serial Interval of COVID-19 among Publicly Reported Confirmed Cases. Emerging Infectious Diseases Journal, 26, 1341-1343. https://doi.org/10.3201/eid2606.200357

[14] Chan, J.F.W., Yuan, S., Kok, K.H., et al. (2020) A familial Cluster of Pneumonia Associated with the 2019 Novel Coronavirus Indicating Person-to-Person Transmission: A Study of a Family Cluster. Lancet, 395, 514-523.

https://doi.org/10.1016/S0140-6736(20)30154-9

[15] Heymann, D.L., Shindo, N. (2020) WHO Scientific and Technical Advisory Group for Infectious Hazards. COVID-19: What is Next for Public Health? Lancet, 395, 542-545. https://doi.org/10.1016/S0140-6736(20)30374-3

[16] Macintyre, C.R. (2020) On a Knife's Edge of a COVID-19 Pandemic: Is Containment Still Possible? Public Health Research and Practice, 30, Article No. 3012000. https://doi.org/10.17061/phrp3012000

[17] Olagniera, D. and Mogensen, H.T. (2020) The Covid-19 Pandemic in Denmark: Big Lessons from a Small Country. Cytokine and Growth Factor Reviews, 53, 10-12. https://doi.org/10.1016/j.cytogfr.2020.05.005

[18] Lipsitch, M. and Dean, N.E. (2020) Understanding COVID-19 Vaccine Efficacy. Science, 370, 763-765. https://doi.org/10.1126/science.abe5938 
[19] Kuwonu, F. (2021) COVID-19: African Countries Scramble for Vaccines. Rich Countries Hoard Available and Future Vaccines, as Poor Countries Wait.

https://www.un.org/africarenewal/magazine/February-2021/covid-19-african-count ries-scramble-vaccines

[20] Prager, F., Wei, D. and Rose, A. (2017) Total Economic Consequences of an Influenza Outbreak in the United States. Risk Analysis, 37, 4-19.

https://doi.org/10.1111/risa.12625

[21] Rose, A., Prager, F., Chen, Z., et al. (2017) Economic Consequence Analysis of Disasters. The E-CAT Software Tool. Springer Eds., USA.

[22] Peak, C.M., Childs, L.M., Grad, Y.H., et al. (2017) Comparing Non-Pharmaceutical Interventions for Containing Emerging Epidemics. Proceedings of the National Academy of Sciences of the United States of America, 114, 4023-4028.

https://doi.org/10.1073/pnas.1616438114

[23] Macintyre, C.R., Costantinso, V. and Kunasekaran, M.P. (2019) Health System Capacity in Sydney, Australia in the Event of a Biological Attack with Smallpox. PLoS ONE, 14, e0217704. https://doi.org/10.1371/journal.pone.0217704 